\title{
Points to consider in the reevaluation and reanalysis of genomic test results: a statement of the American College of Medical Genetics and Genomics (ACMG)
}

\author{
Joshua L. Deignan, PhD ${ }^{1}$, Wendy K. Chung, MD, PhD², Hutton M. Kearney, PhD ${ }^{3}$, \\ Kristin G. Monaghan, $\mathrm{PhD}^{4}$, Catherine W. Rehder, $\mathrm{PhD}^{5}$ and \\ Elizabeth C. Chao, MD'; on behalf of the ACMG Laboratory Quality Assurance Committee
}

\begin{abstract}
Disclaimer: This points to consider document is designed primarily as an educational resource for medical geneticists and other clinicians to help them provide quality medical services. Adherence to this points to consider is completely voluntary and does not necessarily assure a successful medical outcome. This points to consider document should not be considered inclusive of all proper procedures and tests or exclusive of other procedures and tests that are reasonably directed to obtaining the same results. In determining the propriety of any specific procedure or test, the clinician should apply his or her own professional judgment to the specific clinical circumstances presented by the individual patient or specimen.

Clinicians are encouraged to document the reasons for the use of a particular procedure or test, whether or not it is in conformance with this points to consider document. Clinicians also are advised to take notice of the date this points to consider document was adopted, and to consider other medical and scientific information that becomes available after that date. It also would be prudent to consider whether intellectual property interests may restrict the performance of certain tests and other procedures.
\end{abstract}

Keywords: genome; variant; reevaluation; reanalysis; reclassification
Genetics in Medicine (2019) 21:1267-1270; https://doi.org/10.1038/s41436019-0478-1
Reductions in the cost of genomic analyses and the elimination of gene patents for clinical diagnostics have enabled clinical laboratories to provide increasingly comprehensive genetic testing using sequencing, microarrays, and other methods, resulting in the generation of a vast amount of data that then needs to be analyzed. ${ }^{1}$ A significant challenge for clinical laboratory geneticists is the provision of accurate and consistent variant classification. Variant classification has historically been hindered by a lagging recognition of gene-disease associations, as well as a lack of publicly available data (including reference data) from clinical laboratories and other sources. As more clinical laboratories share variant classifications through public databases, discrepancies in variant classification are being better appreciated. $^{2}$

Recognizing the need to improve the existing interpretive framework, the American College of Medical Genetics and Genomics (ACMG) created standards for the interpretation of copy-number variants in 2011 (ref. ${ }^{3}$ ) and published updated standards for the interpretation of sequence variants in 2015 $\left(\right.$ ref. $\left.{ }^{4}\right)$. With increasing amounts of both public and private variant and phenotypic information from affected and unaffected individuals, improved computational algorithms for predicting pathogenicity, and an expanding body of functional studies, the knowledge base for variant classification constantly evolves. ${ }^{5}$ Therefore, variant classification remains a dynamic process, and previously classified variants will frequently benefit from periodic reevaluation.

Furthermore, the phenotype of individuals evolves over time, with relevant features potentially presenting after the initial variant classification has occurred, and recognition of the phenotypic spectrum of a condition may also expand as more individuals with specific genotypes are identified. Therefore, the implications of the variants seen in an individual may not always be clear, and relevant variants may not be reported at the time that the variants are initially classified. As many clinical genomic analyses will fail to yield one or more definitive pathogenic alterations that can

\footnotetext{
${ }^{1}$ Department of Pathology and Laboratory Medicine, University of California Los Angeles, Los Angeles, CA, USA; ${ }^{2}$ Departments of Medicine and Pediatrics, Columbia University, New York, NY, USA; ${ }^{3}$ Department of Laboratory Medicine and Pathology, Mayo Clinic, Rochester, MN, USA; ${ }^{4}$ GeneDx, Gaithersburg, MD, USA; ${ }^{5}$ Department of Pathology, Duke University Medical Center, Durham, NC, USA; ${ }^{6}$ Department of Pediatrics, University of California Irvine, Irvine, CA, USA. Correspondence: Joshua L. Deignan (jdeignan@mednet.ucla.edu) The Board of Directors of the American College of Medical Genetics and Genomics approved this statement on 28 January 2019.
} 
sufficiently explain the clinical indication(s) provided for testing an individual, ${ }^{6-9}$ subsequent reanalyses are critical in the search for a diagnosis as new gene-disease and variantdisease relationships are discovered. ${ }^{10}$

Changes in the interpretation of clinical genomic test results are expected, and health-care providers and the clinical laboratory share a desire for timely reevaluation reflecting emerging new evidence to facilitate appropriate clinical care for the patient and their family. However, periodic reevaluation of DNA variants requires significant time and financial commitment from laboratories and clinicians, for which there is inadequate reimbursement. ${ }^{11}$ As such, reevaluation practices should be designed to maximize clinical impact while minimizing the burden to the laboratory and health-care system. The following Points to Consider should be viewed as informative guidance for physician providers, clinical laboratory geneticists, genetic counselors and, when appropriate, other health-care providers. More specifically, this document is intended to assist laboratories with the development of policies and protocols for variant-level reevaluation and caselevel reanalysis appropriate for their setting and the specific circumstances presented by each clinical scenario.

Variant-level reevaluation involves the interrogation and potential reclassification of previously reported variants. This may be initiated upon external request (by the clinical team or patient), or by the laboratory, either through the systematic review of selected previously reported variants, or through the detection of a previously reported variant in a new case.

Case-level reanalysis involves the review of all variants in an exome or genome, both reported and unreported. Typically, this is intended to identify potentially causative variants after maturation of the knowledge base and/or bioinformatics approaches. Case-level reanalysis may involve only the reevaluation of previously recognized variants, or, when appropriate, may also involve reprocessing the data through the entire analysis pipeline (alignment, variant calling, automated annotation and/or filtering). This process may be initiated upon external request (by the clinical team or patient), or by the laboratory, generally as a periodic approach to unsolved cases or in response to an improvement in bioinformatics tools.

Professional guidance regarding whether the clinical laboratory, ordering clinician, and/or patient are responsible for initiating a request for variant-level reevaluation or caselevel reanalysis is beyond the scope of the present document; these considerations are separately addressed by the ACMG Social, Ethical, and Legal Issues Committee. ${ }^{12}$

\section{GENERAL CONSIDERATIONS}

- Clinical laboratories should have separate policies and protocols for initial variant classification, variant-level reevaluation, and case-level reanalysis, which should be periodically reviewed and updated (as an example, the College of American Pathologists recommends a review of all policies and protocols at least every two years). The laboratory policies should be made available to the ordering provider upon request.

- Clinical laboratories should respond to external requests for variant-level reevaluations or case-level reanalyses in a timely manner and should have policies for addressing those requests.

- Maintaining an up-to-date database of classified variants within the clinical laboratory is critical for the provision of accurate and consistent variant classifications.

- Documentation provided by clinical laboratories (e.g., consent forms, reports) should include the possibility that clinically significant changes in variant classification will occur routinely through new discovery, enhanced clinical correlation, and functional or epidemiologic studies.

- Additional charges/fees may be warranted when the professional services of the clinical laboratory are required for the reevaluation/reanalysis of genomic data. Establishing a mechanism by which these professional services are billable and covered by payers is critical toward ensuring the long-term success of clinical genomic testing.

- In addition to issuing formal reports to clinicians, clinical laboratories should submit classified variants, as well as any reclassifications and updated phenotypic information, to public databases (e.g., ClinVar) on a regular basis. Submissions should be accompanied by a summary of the evidence supporting the classification or reclassification.

\section{CONSIDERATIONS FOR VARIANT-LEVEL REEVALUATION}

- When formulating laboratory policies and protocols for variant-level reevaluation, it is reasonable to prioritize such efforts to maximize the potential clinical impact. For example, classified variants with either highly confident assertions or variants that are not currently thought to be clinically significant (i.e., those that are benign or likely benign) could be reevaluated less frequently than those with uncertain or likely pathogenic classifications.

- Routine reevaluation of a clinical laboratory's entire internal database of variant classifications is likely to be impractical. However, a partial or automated review or a prioritization of variants for re-review may be considered in the following circumstances:

- Availability of a new community resource (e.g., gnomAD)

- Publication and/or adoption of a novel/updated methodology for variant assessment

- Publication of evidence supporting new gene-disease relationships and/or mechanisms of disease

- Upon reevaluation, any variant classified and reported prior to the implementation of the current ACMG variant classification standards should be reevaluated using the 
current ACMG standards.

- Use of an existing variant classification without reevaluation may be considered when the variant has previously been classified without uncertainty (pathogenic, benign) using the laboratory's current protocol.

\section{CONSIDERATIONS FOR CASE-LEVEL REANALYSIS}

- Clinical laboratories are encouraged to request updated clinical and family history information, when available, to facilitate case-level reanalysis of genomic data. Updated information may result in the identification of additional variants that are associated with the indication(s) for testing.

- If significant improvements have been made to the bioinformatics handling of the data (alignment/variant calling and/or the annotation/automated filtering processes), the laboratory should consider (if technically possible) whether case-level reanalysis should also include reprocessing the data through the analysis pipeline to capture any potentially overlooked variants in the initial assessment.

\section{CONSIDERATIONS FOR REPORTING}

- Clinical laboratories should make concerted efforts to prioritize the reporting and communication of any reclassifications that may affect clinical management. For example, a variant of uncertain clinical significance that is reclassified as a likely pathogenic variant should be prioritized as compared to a likely pathogenic variant that is reclassified as a pathogenic variant. Clinical laboratories should also consider the reporting and communication of any variants of uncertain clinical significance that are reclassified to likely benign or benign, as these reclassifications may also have an impact on clinical management.

- For laboratory-initiated variant reevaluations, laboratories should have a policy regarding the release of an updated report when there is a clinically significant change in variant classification, which should include information regarding to whom the updated report is sent when the ordering health-care provider is no longer involved in the individual's care (e.g., the provider relocates, retires, or dies; the patient changes providers).

- For clinician-initiated variant reevaluation requests, laboratories should consider communicating and reporting all updated classifications, even when the initial classification remains unchanged. For reporting purposes, an addendum to the previous report could be issued.

- Reevaluations of variants on the ACMG secondary findings gene list may also have an impact on clinical management, and the reporting and communication of all pathogenic variants (and expected pathogenic variants, if applicable) in a gene that is on the current secondary findings gene list should also be prioritized upon case reanalysis if the patient originally consented to receive this information.

\section{CONSIDERATIONS FOR RETESTING}

- As laboratory technology, processes, and knowledge improve, retesting individuals using new methodologies may be preferable to variant-level reevaluation or caselevel reanalysis. For example, genome sequencing may be indicated for an individual with nondiagnostic results by exome sequencing.

- When considering reanalysis versus retesting, some important factors to consider include:

- The time elapsed since the previous testing occurred

- Improvements in technology/chemistry (e.g., new methods for DNA capture and sequencing)

- Bioinformatics advancements

- New information regarding the genetic etiology of a condition

- Any additional patient phenotypes or family history that developed in the interim

\section{CONCLUSION}

Clinical laboratories should have policies and protocols for variant-level reevaluation and case-level reanalysis, and these policies and protocols should keep pace with any new developments in population databases, genomic curation, bioinformatics, and electronic health records. The efforts required to provide accurate and consistent variant classifications will be bolstered in the future by automation and changes in reimbursement. Ensuring that clinical laboratories can recoup the expense of this effort will be critical as the field evolves.

\section{DISCLOSURE}

All authors serve as directors in clinical laboratories that perform a breadth of genetic and genomic analyses on a fee-for-service basis. K.G.M. is an employee of GeneDx, a wholly owned subsidiary of OPKO Health, Inc.

Publisher's note: Springer Nature remains neutral with regard to jurisdictional claims in published maps and institutional affiliations.

\section{REFERENCES}

1. Rehm HL, Bale SJ, Bayrak-Toydemir P, et al. ACMG clinical laboratory standards for next generation sequencing. Genet Med. 2013;15:733-747.

2. Harrison SM, Dolinsky JS, Chen W, et al. Scaling resolution of variant classification differences in ClinVar between 41 clinical laboratories through an outlier approach. Hum Mutat. 2018;39:1641-1649.

3. Kearney HM, Thorland EC, Brown KK, et al. American College of Medical Genetics standards and guidelines for interpretation and reporting of 
postnatal constitutional copy number variants. Genet Med 2011;13:680-685.

4. Richards S, Aziz N, Bale S, et al. Standards and guidelines for the interpretation of sequence variants: a joint consensus recommendation of the American College of Medical Genetics and Genomics and the Association for Molecular Pathology. Genet Med. 2015;17:405-424.

5. Ghosh R, Oak N, Plon SE. Evaluation of in silico algorithms for use with ACMG/AMP clinical variant interpretation guideline. Genome Biol. 2017; 18:225.

6. Lee $\mathrm{H}$, Deignan JL, Dorrani $\mathrm{N}$, et al. Clinical exome sequencing for genetic identification of rare Mendelian disorders. JAMA. 2014;312:1880-1887.

7. Yang Y, Muzny DM, Reid JG, et al. Clinical whole-exome sequencing for the diagnosis of Mendelian disorders. N Engl J Med. 2013;369:1502-1511.

8. Retterer K, Juusola J, Cho MT, et al. Clinical application of whole-exome sequencing across clinical indications. Genet Med. 2016;18:696-704.
9. Farwell KD, Shahmirzadi L, El-Khechen D, et al. Enhanced utility of familycentered diagnostic exome sequencing with inheritance model-based analysis: results from 500 unselected families with undiagnosed genetic conditions. Genet Med. 2015;17:578-586.

10. Wenger AM, Guturu H, Bernstein JA, Bejerano $G$, et al. Systematic reanalysis of clinical exome data yields additional diagnoses: implications for providers. Genet Med. 2017;19:209-214.

11. O'Daniel JM, McLaughlin HM, Amendola LM, et al. A survey of current practices for genomic sequencing test interpretation and reporting processes in US laboratories. Genet Med. 2017:19:575-582.

12. David KL, Best RG, Brenman LM, et al. Patient re-contact after revision of genomic test results: points to consider-a statement of the American College of Medical Genetics and Genomics (ACMG). Genet Med. 2018 Dec 22; https://www.nature.com/articles/s41436-018-0391-z [Epub ahead of print]. 